Revue de droit comparé du travail et de la sécurité sociale

$3 \mid 2020$

La Directive 2019/1158 du 20 juin 2019 concernant l'équilibre entre vie personnelle et vie privée des parents et des aidants

\title{
La directive de 2019 sur l'équilibre entre vie professionnelle et vie privée : une nouvelle étape franchie
}

\section{Eugenia Caracciolo di Torella}

\section{OpenEdition}

Journals

Édition électronique

URL : https://journals.openedition.org/rdctss/942

DOI : $10.4000 /$ rdctss.942

ISSN : 2262-9815

Éditeur

Centre de droit comparé du travail et de la sécurité sociale

Édition imprimée

Date de publication : 1 novembre 2020

Pagination : 8-19

ISSN : 2117-4350

Référence électronique

Eugenia Caracciolo di Torella, « La directive de 2019 sur l'équilibre entre vie professionnelle et vie privée : une nouvelle étape franchie », Revue de droit comparé du travail et de la sécurité sociale [En ligne], 3 | 2020, mis en ligne le 01 novembre 2021, consulté le 11 novembre 2021. URL : http:// journals.openedition.org/rdctss/942; DOI : https://doi.org/10.4000/rdctss.942

Revue de droit comparé du travail et de la sécurité sociale est mise à disposition selon les termes de la Licence Creative Commons Attribution - Pas d'Utilisation Commerciale - Pas de Modification 4.0 International. 


\title{
LA DIRECTIVE DE 2019 SUR L'ÉQUILIBRE ENTRE VIE PROFESSIONNELLE ET VIE PRIVÉE : UNE NOUVELLE ÉTAPE FRANCHIE
}

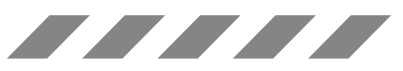

\begin{abstract}
This paper offers an early analysis of the recently adopted 2019 directive on Work-Life Balance for Parents and Carers. In particular, it focuses on the 4 substantive rights included in the directive : parental leave, paternity leave, carers' leave and the right to ask for flexible working arrangements. The paper aims to highlight the directive's successes and to identify the areas that still need improvement. It concludes that this instrument is certainly a bold and innovative development and a welcome acknowledgment of the needs of working carers. Its aims, however, are only partially reached, as such it is one more step along way but certainly not the final one.
\end{abstract}

KEYWORDS: Work-life Balance, Parental Leave, Flexible Working Arrangements, Care, Children, Dependants.

\section{RÉSUMÉ}

Cet article propose une analyse de la directive de 2019 qui a récemment été adoptée sur l'équilibre entre la vie professionnelle et la vie privée pour les parents et les aidants. En particulier, il se concentre sur 4 droits consacrés par la directive : le congé parental, le congé de paternité, le congé pour soignants et le droit de demander des modalités de travail flexibles. Cette étude vise à mettre en évidence les avancées de la directive et à identifier les domaines qui doivent être encore améliorés. Ce nouvel instrument juridique est sans doute audacieux et innovant notamment parce qu'il reconnait des besoins exprimés par les aidants qui travaillent. Cependant, les objectifs ne sont que partiellement atteints si bien qu'il s'agit d'une étape de plus, certainement pas la dernière dans la recherche d'un équilibre entre vie professionnelle et vie privée.

MOTS CLÉS: Équilibre entre vie professionnelle et vie privée, congé parental, formules souples de travail, soins, enfants, personnes à charge. 
n août 2019, la directive tant attendue sur l'équilibre entre vie professionnelle et vie privée des parents et aidants (dénommée " directive WLB » au Royaume-Uni) est entrée en vigueur ${ }^{1}$. Cette directive peut être considérée comme l'enfant né de la tentative avortée de modifier la directive sur les travailleuses enceintes en $2015^{2}$. Cependant, son origine remonte à bien plus loin : non seulement à la tentative partiellement concluante - d'élaborer un paquet « vie professionnelle et vie privée » en $2008^{3}$, mais aussi et surtout à l'époque même où l'objectif de " conciliation entre vie professionnelle et vie familiale » fut mis à l'ordre du jour en $1972^{4}$. Entre 1972 et 2019, de nombreuses étapes ont été franchies en termes de législation, d'interprétation judiciaire et d'initiatives politiques 5 . L'adoption du Socle européen des droits sociaux (SEDS) a constitué un moment décisif dans ce processus ${ }^{6}$. S'il ne s'agit pas d'un instrument contraignant, il a toutefois été décrit comme " une direction à suivre pour renouer avec la convergence vers le haut des normes sociales, dans un monde du travail en mutation " $^{7}$. II a déjà prouvé son immense potentiel : en effet, dans son cadre, la Commission a proposé un "Nouveau départ pour soutenir l'équilibre entre vie professionnelle et vie privée des parents et des aidants $»^{8}$ qui a finalement abouti à la directive.

1 Directive (UE) 2019/1158 du Parlement européen et du Conseil du 20 juin 2019 relative à l'équilibre entre vie professionnelle et vie privée des parents et aidants, abrogeant la directive 2010/18/CE du Conseil JO [2019] L188/79.

2 Proposition de directive modifiant la directive 92/85/CEE du Conseil concernant la mise en œuvre de mesures visant à promouvoir l'amélioration de la sécurité et de la santé des travailleuses enceintes, accouchées ou allaitantes au travail, COM(2008)637. Elle a été rejetée par le Conseil en décembre 2010, puis supprimée par la Commission car elle était considérée comme une « charge administrative ». Voir Commission européenne, "Faciliter la vie des parents : la Commission retire sa proposition en attente sur le congé de maternité et ouvre la voie à une nouvelle approche ", Bruxelles, 1 er juillet 2015 (https://ec.europa.eu/commission/presscorner/ detail/en/IP 15 5287).

3 Le paquet «vie professionnelle et vie privée » proposé par la Commission européenne (MEMO/08/603 du 3 octobre 2008) a été adopté quelques semaines avant que la crise de 2008 ne s'abatte sur l'UE. Le paquet « vie professionnelle et vie privée » comprenait un certain nombre de documents, dont deux propositions de loi visant à réviser les directives existantes : la directive sur les travailleuses enceintes $(\mathrm{COM}(2008) 637)$ et la directive sur les travailleurs indépendants (Proposition de directive concernant l'application du principe de l'égalité de traitement entre hommes et femmes exerçant une activité indépendante et abrogeant la directive 86/613/CEE, $\operatorname{COM}(2008) 636)$. Seule cette dernière a été retenue.

4 Programme d'action sociale 1974, CE Bull Supp 2/74.

5 E. Caracciolo di Torella et A. Masselot, Reconciling Work and Family Life in EU Law and Policy, Palgrave, 2010.

6 Proclamation interinstitutionnelle relative au Socle européen des droits sociaux, JO [2017] C428/10.

7 Commission européenne, "Suivi de la mise en œuvre du socle européen des droits sociaux », $\operatorname{COM}(2018) 130$, p. 2.

8 Communication de la Commission, "Initiative visant à promouvoir l'équilibre entre vie professionnelle et vie privée des parents et aidants qui travaillent ", (COM 92017) 252 final (https://ec.europa.eu/info/law/better-regulation/initiatives/com-2017-253 en). Voir également E. Caracciolo di Torella, «An Emergency Right to Care in the EU: A New Start to Support Work-Life Balance for Parents and Carers », ERA Forum, vol. 18, 2017, p. 187. 
La présente étude, structurée autour de trois parties, vise à offrir une première analyse de la directive, à souligner ses points forts et à identifier les domaines à améliorer. Dans un premier temps, cet article présente brièvement le contexte dans lequel la directive a été adoptée et ses objectifs (I), avant de procéder à une analyse du champ d'application personnel de la directive (II), puis du champ d'application matériel (III). Cette dernière partie examine en particulier les quatre principaux droits inclus dans la directive, mais aussi ceux qui n'y figurent pas. Sur la base de cette réflexion, on peut considérer que la directive WLB est une avancée audacieuse et innovante et, à ce jour, l'instrument le plus complet de son genre. Ses objectifs, cependant, ne sont que partiellement atteints ; c'est pourquoi elle constitue une nouvelle étape, mais certainement pas la dernière. Pour révéler tout son potentiel, la directive aura vraisemblablement besoin du soutien proactif de la Cour de justice de l'Union européenne (CJUE).

\section{I - LA DIRECTIVE SUR L'ÉOUILIBRE ENTRE VIE PROFESSIONNELLE ET VIE PRIVÉE : CONTEXTE ET OBJECTIFS}

La directive sur l'équilibre entre vie professionnelle et vie privée marque indéniablement un progrès bienvenu dans ce domaine, en ce sens qu'elle témoigne d'une reconnaissance claire des besoins des aidants qui travaillent. Cette directive est arrivée à un moment où, après quasiment une décennie de politiques d'austérité suite à la crise financière de 2008, se dessinait une volonté claire de renforcer la dimension sociale de l'UE pour améliorer les conditions de travail et de vie des citoyens et, en particulier, des femmes. Le législateur était en effet bien conscient qu'en raison de leurs responsabilités familiales, les femmes restent sous-représentées sur le marché du travail et qu'elles sont toujours nettement moins bien rémunérées que les hommes ${ }^{9}$. Cette directive a été l'une des premières mesures adoptées pour mettre en œuvre les principes du $\operatorname{SEDS}^{10}$. Ce document contient 20 principes couvrant des questions de droit du travail telles que «le droit à un salaire équitable [et] à un traitement juste et équitable», mais aussi des questions plus larges de politique sociale.

Le principe 9 est particulièrement pertinent dans ce contexte : "Les parents et les personnes ayant des responsabilités familiales ont droit à des congés appropriés, à des régimes de travail flexibles et à un accès à des services de garde d'enfants. Les femmes et les hommes doivent bénéficier d'un accès égal aux congés spéciaux afin de s'acquitter de leurs responsabilités familiales et sont encouragés à les utiliser de manière équilibrée ».

Non seulement cette disposition a contribué à accroître la visibilité des aidants et des parents qui travaillent, mais elle a également élargi le discours. Les bases juridiques qui sous-tendent cette directive en témoignent :

- I'article 153, paragraphe 1, du TFUE, prévoit que I'Union soutient et complète l'action des États membres dans le domaine de l'égalité ;

9 En 2017, l'écart du taux d'emploi entre les femmes et les hommes s'élevait à $11 \%$, avec 68,2\% des femmes dans I'UE ayant un emploi contre 79,2\% des hommes. L'écart de rémunération entre les hommes et les femmes dans l'UE s'élève à $16 \%$ et n'a que très peu évolué au cours des dix dernières années. Cela signifie que les femmes gagnent en moyenne $16 \%$ de moins par heure que les hommes. Voir Commission européenne : https://ec.europa.eu/info/policies/justice-andfundamental-rights/gender-equality/equal-pay/gender-pay-gap-situation-eu en

10 Voir également la directive 2019/1152 du Parlement européen et du Conseil du 20 juin 2019 relative aux conditions de travail transparentes et prévisibles dans I'Union européenne, [2019] JO L186/105, dont il est question plus en détail dans cet article. 


\section{ANALYSE DE LA DIRECTIVE 2019/1158}

- l'article 3, paragraphe 3, du TUE, fait expressément référence à la promotion de l'égalité ; - les articles 23 et 33 de la Charte européenne des droits fondamentaux sont consacrés à l'égalité entre les femmes et les hommes d'une part, et à la vie familiale et vie professionnelle d'autre part.

On peut également relever des références expresses à la Convention des Nations unies de 2006 sur les droits des personnes handicapées, à la Convention des Nations unies de 1989 sur les droits de l'enfant, ainsi bien sûr qu'au Rapport sur le SEDS.

Pour la première fois, le législateur européen aborde la question de l'équilibre entre vie professionnelle et vie privée. Ainsi, il offre un angle d'analyse différent des droits existants : il ne s'agit plus seulement d'un problème qui concerne les femmes ou (à la limite) les parents, mais d'un problème qui peut (et va) toucher la plupart des travailleurs.

Partant du constat que « l'équilibre entre vie professionnelle et vie privée reste un défi considérable $»^{11}$, la directive a pour objectif principal de prendre en compte l'évolution des besoins et la diversité des responsabilités familiales et des aidants, en modernisant un cadre mis en place dans les années 1990 qui n'a pas vraiment changé depuis. Plus précisément, elle cherche à y parvenir en étendant sa protection à un plus large éventail de familles et d'individus, en encourageant les hommes à assumer davantage de responsabilités familiales, et en réitérant le message selon lequel « la situation d'un homme salarié et celle d'une femme salariée sont comparables en ce qui concerne l'éducation des enfants ${ }^{12}$, ainsi qu'en développant l'utilisation de formules souples de travail.

\section{II - LE CHAMP D'APPLICATION PERSONNEL DE LA DIRECTIVE}

Au moment de sa rédaction, la directive sur le congé parental garantissait déjà que certains droits liés à la famille s'appliquent à tous les travailleurs " y compris aux travailleurs à temps partiel, aux travailleurs à durée déterminée ou aux personnes en contrat ou en relation de travail avec une entreprise de travail intérimaire ${ }^{13}$. La directive WLB va encore plus loin dans la mesure où, selon son article 2, elle s'applique à tous les travailleurs ayant un contrat ou une relation de travail tel que défini par les États membres et « compte tenu de la jurisprudence de la Cour de justice ${ }^{14}$. Cette référence est particulièrement importante car elle reconnaît le risque selon lequel, si l'on laisse aux États membres le soin de déterminer le champ d'application personnel de la directive, ce dernier pourrait être interprété de manière restrictive ce qui irait à l'encontre de son objectif.

11 Considérant 10, directive 2019/1158. Pour un aperçu de la situation, voir A. Masselot, Rapport thématique "Family leave: enforcement of the protection against dismissal and unfavourable treatment ", 2018 (http://dx.doi.org/10.2838/39810).

12 Affaire C-222/14, Konstantinos Maïstrellis contre Ypourgos Dikaiosynis, Diafaneias kai Anthropinon Dikaiomaton, EU:C:2015:473, § 47.

13 Clause 1(3) Directive 2010/18/UE du Conseil portant application de l'accord-cadre révisé sur le congé parental conclu par Business Europe, I'UEAPME, le CEEP et la CES et abrogeant la directive 96/34/CE [2010] JO L 68/13.

14 Directive 2010/18 du Conseil. 
L'article 2 s'inspire de l'article 1, paragraphe 2, de la directive sur les conditions de travail transparentes et prévisibles ${ }^{15}$ (directive TPWC), également adoptée dans le cadre du SEDS. Cet instrument répond à la flexibilisation croissante du marché du travail et étend les normes minimales existantes à de nouvelles formes d'emploi. Il vise ainsi à protéger un large éventail de travailleurs, en particulier les plus vulnérables ${ }^{16}$. En outre, le considérant 8 de la directive TPWC explique que si les critères fixés par la CJUE sont remplis, les travailleurs tels que les « les travailleurs domestiques, les travailleurs à la demande, les travailleurs intermittents, les travailleurs effectuant un travail basé sur des "chèques", les travailleurs des plateformes, les stagiaires et les apprentis » pourraient bénéficier de cette disposition.

Le fait que l'article 2 de la directive reprenne la formulation de l'article 1, paragraphe 2, de la directive TPWC, démontre pour le moins qu'il existe une vraie prise de conscience de la mutation du marché du travail et de la vulnérabilité de nombreux travailleurs. II reste cependant à apprécier la manière dont cette disposition sera interprétée ${ }^{17}$.

\section{III - LE CHAMP D'APPLICATION MATÉRIEL DE LA DIRECTIVE}

La directive abroge l'actuelle directive sur le congé parental ${ }^{18}$, élargit et renforce les droits existants. Plus précisément, elle introduit deux droits phares, le congé de paternité (A) et le congé pour les aidants naturels (C) ; elle renforce deux autres droits, le congé parental (B) et le droit de demander des formules souples de travail (D). Ce faisant, elle prévoit également des dispositions spécifiques ouvrant la voie à de nouvelles protections pour garantir l'efficacité de ces droits (E).

Néanmoins, il est curieux de constater que la directive n'aborde pas la situation des mères. D'une part, les amendements proposés à la directive sur les travailleuses enceintes ayant provoqué une impasse au sein du Conseil et conduit à son retrait, il peut être compréhensible que ces mêmes amendements n'aient pas pu être proposés à nouveau aussi rapidement ${ }^{19}$. D'autre part, tout instrument relatif à l'équilibre entre vie professionnelle et vie privée ne saurait être complet sans tenir compte des mères. Ainsi, dans l'ensemble, cela semble être une occasion manquée et le retrait de la proposition de directive aurait pu être utilisé comme un tremplin pour introduire un cadre plus progressif pour traiter les questions d'équilibre entre vie professionnelle et vie privée. Ce cadre aurait pu prévoir des situations de plus en plus courantes, telles que la FIV et la maternité de substitution, des dispositions spécifiques sur l'allaitement, ainsi qu'une protection renforcée en matière de licenciement pour les femmes revenant d'un congé de maternité. Au lieu de cela, les droits des femmes enceintes et des nouvelles mères continuent d'être fixés par d'autres

15 Directive 2019/1152. Voir B. Bednarowicz, "Delivering on the European Pillar of Social Rights : The New Directive on Transparent and Predictable Working Conditions in the European Union », Industrial Law Journal, 2019, p. 604.

16 Voir aussi E. Chieregato, «A Work-Life Balance for all? Assessing the Inclusiveness of EU Directive 2019/1158 ", Journal of Comparative Labour Law and Industrial Relations, 2020, n³6, p. 1.

17 E. Chieregato, ibid. Voir également le considérant 17 de la directive (UE) 2019/1158, et B. Bednarowicz, «Delivering on the European Pillar of Social Rights: The New Directive on Transparent and Predictable Working Conditions in the European Union », op. cit.

18 Directive 2010/18/UE du Conseil.

19 Supra note 3. 


\section{ANALYSE DE LA DIRECTIVE 2019/1158}

instruments, notamment la directive sur l'égalité de traitement ${ }^{20}$ et la directive sur les travailleuses enceintes, cette dernière ayant près de 30 ans $^{21}$.

\section{A - DROITS DES PÈRES}

Le congé de paternité est expressément prévu à l'article 4 qui accorde le droit de prendre au moins dix jours ouvrables lors de la naissance de l'enfant. Si de nombreux États membres prévoient déjà ce droit, c'est la toute première fois que le législateur européen le reconnaît comme un droit individuel. Parmi les précédentes tentatives de réglementation de la position des pères, on peut citer la directive modifiée sur l'égalité de traitement ${ }^{22}$ ainsi que la directive de refonte ${ }^{23}$. Ces instruments prévoyaient simplement que le même niveau de protection appliqué au congé de maternité soit également étendu aux congés de paternité et d'adoption, à condition que les États membres aient déjà introduit de telles dispositions dans leur droit national. Autrement dit, les droits des pères étaient jusqu'à présent considérés comme une option devant être examinée par les États membres.

En introduisant l'article 4, la directive souhaite encourager les pères à s'impliquer activement. Elle entend faire clairement comprendre que les deux parents sont tenus de s'occuper de leurs enfants au même titre ${ }^{24}$ et vise à reconnaître la diversité des formes familiales. Comme pour le congé de maternité, il s'agit d'un droit inconditionnel : il ne peut être soumis à une période de qualification professionnelle ou à une durée d'ancienneté, et les employeurs ne peuvent ni le refuser, ni le reporter. De plus, comme pour le congé de maternité, le congé de paternité est rémunéré au moins au niveau du congé de maladie ${ }^{25}$.

Si le fait de mettre l'accent sur les pères est bienvenu, et plus que nécessaire, l'article 4 ne place pas nécessairement les parents dans une position d'égalité lorsqu'il s'agit de s'occuper des enfants. Tout d'abord, la mère continue à bénéficier d'une période de congé beaucoup plus longue. Par ailleurs, comme ce droit doit être pris "lors de la naissance ", les pères en useront en pratique en même temps que la mère, et ne seront donc pas seuls à s'occuper de l'enfant. De plus, bien qu'ils soient tous deux rémunérés au même niveau que les indemnités de maladie, il n'est pas rare que les États membres complètent les indemnités de maternité. D'autre part, la directive permet aux États membres de soumettre le droit aux indemnités à des périodes d'emploi antérieures, ce qui, bien que cela ne

20 La directive 2006/54/CE relative à la mise en œuvre du principe de l'égalité des chances et de l'égalité de traitement entre hommes et femmes en matière d'emploi et de travail (refonte) [2006] JO L 204/23.

21 La directive 92/85/CEE du Conseil concernant la mise en œuvre de mesures visant à promouvoir l'amélioration de la sécurité et la santé au travail des travailleuses enceintes, accouchées ou allaitantes.

22 Art. 2, § 7, de la directive 2002/73/CE relative à la mise en œuvre du principe de l'égalité des chances et de l'égalité de traitement entre hommes et femmes en matière d'emploi et de travail [2002] JO L 269/15 stipule qu'« il est (...) sans préjudice de la faculté dont disposent les États membres de reconnaître des droits distincts au congé de paternité et/ou d'adoption ».

23 Art. 16 directive 2006/54/CE relative à la mise en œuvre du principe de l'égalité des chances et de l'égalité de traitement entre hommes et femmes en matière d'emploi et de travail (refonte) [2006] JO L 204/23 concerne le congé de paternité et d'adoption et confirme les dispositions de la directive 2002/73/CE sur les pères dans des termes approximativement identiques.

24 Considérant 19, directive 2019/1158.

25 Art. 8. 
puisse pas dépasser six mois, peut limiter le nombre de pères qui peuvent bénéficier de ce droit. En outre, les indemnités de maladie varient considérablement en Europe ${ }^{26}$ et ne garantissent pas partout un revenu adéquat.

À cet égard, l'affaire Gassmayr, qui a trait à la directive sur les travailleuses enceintes, constitue une référence utile : la Cour a estimé que le faible niveau de rémunération ne pouvait pas compromettre l'objectif de la directive ${ }^{27}$. Enfin, le congé de paternité n'est pas obligatoire, contrairement aux deux semaines de congé de maternité avant et/ou après l'accouchement ${ }^{28}$. Ainsi, le message selon lequel les mères sont les principales responsables de la famille reste fort et évident.

En étendant expressément ce droit aux seconds parents et en l'accordant indépendamment de la situation matrimoniale ou familiale telle que définie dans le droit national, l'article 4 reconnaît la pluralité des structures familiales dans la société actuelle: le congé de paternité devient un droit accessible également aux familles homosexuelles et fluides de genre. Toutefois, cela ne couvre pas nécessairement les familles monoparentales qui sont sans doute les plus vulnérables. La référence au « deuxième parent équivalent (...) dans la mesure où il est reconnu par le droit national » laisse la porte ouverte à d'éventuelles disparités.

L'article 4 aurait pu aller plus loin en précisant que dans certains cas, par exemple si le père ne vit pas avec la mère ou si la mère est un parent célibataire, elle peut désigner un ami ou un parent qui vit à proximité afin de bénéficier du congé.

\section{B - CONGÉ PARENTAL}

L'article 5 prévoit un droit individuel au congé parental. Ce droit n'est pas nouveau ${ }^{29}$, mais la directive vise à le renforcer de plusieurs manières afin d'encourager les pères à s'occuper davantage de leurs enfants et d'élargir le nombre de familles qui peuvent I'utiliser. Tout d'abord, la proportion de congés non transférables entre les parents est portée d'un à deux mois, sur la base du principe «à prendre ou à laisser ». À l'heure actuelle, il est prouvé que la plupart des pères transfèrent une part importante de leurs droits aux mères $^{30}$. Ensuite, cet article permet de prendre le droit de manière flexible, le manque de flexibilité pouvant dissuader les pères à prendre leur congé ${ }^{11}$. En outre, il prévoit que les États membres prennent des dispositions pour adapter le congé parental aux besoins des parents adoptifs, des parents handicapés et des parents d'enfants handicapés ou

26 Commission européenne, "Paternity and parental Leave policies across the European Union. Assessment of current provisions. European Commission », 2018 : https://op.europa.eu/en/ publication-detail/-/publication/ a8464ad8-9abf-11e8-a408-01aa75ed71a1/language-en

27 Affaire C-194/08, Gassmayrv Bundesminister für Wissenschaft und Forschung, UE:C:2010:386. Voir en particulier les $\S 61,68,72$ et 73 .

28 Art. 8, § 2, de la directive 92/85/CEE du Conseil.

29 Actuellement, il est prévu dans la directive 2010/18/UE du Conseil et avant cela dans la directive 96/34 [2010] JO L68/13.

30 Commission européenne, "Paternity and parental leave policies across the European Union Assessment of current provision », 2018 ; Eurofound Promoting Uptake of Parental and Paternity Leave Among Fathers in the European Union, 2015 : https://www.eurofound.europa.eu/ publications/customised-report/2015/working-conditions-industrial-relations/promoting-uptakeof-parental-and-paternity-leave-among-fathers-in-theeuropean-union

31 Eurofound, ibid. 


\section{ANALYSE DE LA DIRECTIVE 2019/1158}

atteints d'une maladie de longue durée. Cela permettra de répondre aux besoins d'un plus grand nombre de familles et donc de garantir que davantage de personnes auront accès au congé. Enfin, pour la première fois, la directive prévoit qu'une compensation ou une allocation doit être associée au congé parental.

Cette disposition est complétée par le considérant 29 qui précise que, pour inciter les travailleurs - en particulier les hommes - à prendre les périodes de congé, la compensation ou l'allocation devrait être fixée à un " niveau adéquat». Le considérant 31 indique en outre que, le congé parental entraînant souvent une perte de revenu pour la famille, le parent gagnant le salaire le plus élevé (généralement des hommes) ne pourra faire usage de son droit que s'il est « suffisamment bien rémunéré, en vue de permettre un niveau de vie décent $»^{32}$.

Les propositions antérieures visant à fixer la limite au niveau des indemnités de maladie (comme suggéré dans la proposition initiale de la Commission) ou à $78 \%$ du salaire brut (comme suggéré par le Parlement européen), ont été rejetées par le Conseil ${ }^{33}$. La version finale de la directive laisse la décision aux États membres. Il va sans dire que cela laisse la porte ouverte à des niveaux très différents et, dans le meilleur des cas, cela peut créer des disparités entre les États membres, et dans le pire des cas, cela peut simplement ne pas encourager les pères à prendre ce congé. II est regrettable que l'article 5 ne soit pas plus précis, et il sera crucial de voir comment cette exigence sera interprétée par la CJUE.

\section{C - CONGÉ POUR LES AIDANTS}

Parmi les droits introduits par la directive, le plus marquant est sans doute le congé pour les aidants, prévu à l'article 6. Cet article accorde aux travailleurs un droit individuel de prendre un congé de cinq jours ouvrables par an pour s'occuper d'un proche (paragraphe 1) et propose que les États membres puissent en prolonger la durée " selon la personne qui a besoin de soins ou d'assistance, ou selon le cas » (paragraphe 2). Le considérant 27 souligne en outre la portée de ce droit qui est d' "offrir aux hommes et aux femmes de plus grandes possibilités de rester dans la vie active ».

De prime abord, il s'agit d'une initiative sans précédent susceptible de changer le paradigme. C'est la première fois que le législateur européen prend en compte les besoins des aidants qui travaillent, autres que les parents. En reconnaissant que les responsabilités familiales ne se limitent pas à la garde d'enfants, du moins en principe, l'article 6 prévoit une approche fondée sur le cycle de vie. Au moment de la rédaction, la seule disposition de I'UE que (certains) aidants peuvent utiliser n'est rien d'autre qu'un filet de sécurité 34 : le droit à un congé pour cause de force majeure, c'est-à-dire une période de congé sans solde pour s'occuper en urgence d'un membre de sa famille en cas de maladie ou d'accident qui rend indispensable la présence immédiate du travailleur ${ }^{35}$. Plus important encore, l'article 6 fournit enfin une base juridique sur laquelle le législateur européen peut agir.

32 Italique ajouté.

33 Á. Oliveira, M. De la Corte-Rodríguez et F. Lutz, «The New Directive on Work-Life Balance : Towards a New Paradigm of Family Care and Equality? », European Law Review, 2020, p. 295.

34 E. Caracciolo di Torella et A. Masselot, Caring Responsibilities in European law and Policy. Who Cares?, Routledge, 2020, p. 122.

35 Actuellement dans la clause 7(1) de la directive 2010/18/UE sur le congé parental et maintenu dans l'art. 7 de la directive 2019/1158. 
Dans la pratique cependant, cette disposition ne devrait pas entraîner de changements majeurs dans la vie de la plupart des aidants : de nombreux ordres juridiques nationaux contiennent déjà cette disposition.

L'article 6 présente également quelques faiblesses particulières. Tout d'abord, il repose sur une définition très restrictive de la notion d'aidant. L'article 3, point d), précise qu'il s'agit d'un « travailleur qui fournit des soins ou un soutien personnels à un membre de sa famille ou à une personne vivant sous le même toit que lui et qui a besoin de soins ou d'un soutien importants pour des raisons médicales graves ${ }^{36}$. Cette définition est susceptible d'exclure de nombreuses situations de prise en charge. D'autant que l'article 3, point e), précise qu'un membre de la famille est un enfant, un parent, un conjoint ou un partenaire civil d'un travailleur dans le cas où le droit national envisage de tels partenariats civils. Bien que le considérant 27 « encourage » les États membres à accorder ce droit « aux autres membres de la famille, tels que les grands-parents et les frères et sœurs ", il n'est pas adapté pour couvrir toutes les situations où des soins sont nécessaires.

En outre, la durée limitée ( 5 jours) du droit n'a pas d'utilité pour ceux qui doivent s'occuper régulièrement d'un proche de manière continue. Enfin, le fait que le droit ne soit pas rémunéré ${ }^{37}$ envoie non seulement un mauvais message quant à la valeur des soins, mais il risque également de ne pas encourager les hommes à utiliser le congé et donc de maintenir le statu quo.

En résumé, si l'article 6 ne prévoit pas le cadre politique en matière de responsabilités familiales dont on a pourtant tant besoin, si ce n'est un « droit aux soins », il pourrait toutefois s'avérer être une étape vers sa mise en place.

\section{D - FORMULES SOUPLES DE TRAVAIL}

L'article 9 renforce les dispositions relatives aux formules souples de travail. II s'agit sans doute de la mesure la moins novatrice parmi celles qui ont été introduites. Au fil des ans, plusieurs instruments juridiques visant à réglementer la flexibilité sur le marché du travail, tels que la directive sur le travail à temps partiel ${ }^{38}$, la directive sur les contrats à durée déterminée ${ }^{39}$ et la directive sur le travail intérimaire, ont été adoptés ${ }^{40}$. Ces mesures n'ont pas été conçues dans un souci d'équilibre entre vie professionnelle et vie privée, mais plutôt pour des raisons économiques.

Pourtant, il n'est pas surprenant que les parents et les aidants y aient eu recours en raison du manque de services de soins abordables et/ou de qualité ainsi que de l'incapacité

36 Italique ajouté.

37 La proposition initiale prévoyait que ces journées soient rémunérées, voir la proposition de directive du Parlement européen et du Conseil relative à l'équilibre entre vie professionnelle et vie privée des parents et aidants, COM(2017) 253 final.

38 Directive 97/81/CE du Conseil du 15 décembre 1997 concernant l'accord-cadre sur le travail à temps partiel conclu par I'UNICE, le CEEP et la CES - Annexe : Accord-cadre sur le travail à temps partiel, JO [1998] L14/9.

39 Directive 99/70/CE du Conseil du 28 juin 1999 concernant l'accord-cadre CES, UNICE et CEEP sur le travail à durée déterminée, JO [1999] L175/43, rectificatif au JO [1999] L244/64.

40 Directive 2008/104/CE du Parlement européen et du Conseil du 19 novembre 2008 relative au travail intérimaire, JO [2008] L327/9. 


\section{ANALYSE DE LA DIRECTIVE 2019/1158}

qui en découle à travailler à temps plein ou à occuper un emploi rémunéré ${ }^{41}$. II n'est pas non plus étonnant que ces "parents et aidants » soient principalement des femmes pour lesquelles «la double obligation de travail rémunéré et non rémunéré rend essentielle la perméabilité de la frontière entre le domicile et le marché du travail ». La flexibilité du travail est le seul moyen pour de nombreuses femmes de franchir cette frontière ${ }^{42}$. Depuis 2010, la directive sur le congé parental accorde aux parents qui reviennent d'un congé parental le droit de demander un aménagement de leurs horaires de travail ${ }^{43}$.

L'article 9 va plus loin et lie la flexibilité au concept d'équilibre entre vie professionnelle et vie privée. Ce faisant, il remet en question l'approche traditionnelle de l'UE en matière de formules souples de travail : celles-ci ne sont plus considérées comme motivées par des raisons économiques ou comme étant spécifiquement nécessaires aux parents, mais comme des formules destinées à toute personne ayant des responsabilités familiales. Plus précisément, l'article 9 établit que les travailleurs ayant des enfants d'un âge spécifié par les États membres et, en tout état de cause, pas plus de 8 ans, ainsi que les aidants (tels que définis par la directive) « ont le droit de demander des formules souples de travail dans le but de s'occuper de leurs proches ». II indique trois moyens pour y parvenir : la réduction du temps de travail, la modification des rythmes de travail ainsi que les modalités de travail à distance ou le télétravail. La référence au travail à distance est une nouveauté bienvenue dans la directive. Autre élément important : une formule souple de travail peut être convenue pour une période limitée ; dans ce cas, le travailleur a le droit de revenir à son rythme de travail initial. Il a également le droit de demander à revenir à son rythme de travail initial si les circonstances changent. Jusqu'à présent, une fois qu'un aménagement de travail avait été convenu, il était définitif.

Le droit de demander des formules souples de travail est également renforcé par la directive sur les conditions de travail transparentes et prévisibles ${ }^{44}$, adoptée le même jour que la directive WLB. La directive TPWC vise à créer des relations de travail plus sûres face à la multiplication des formes de travail flexibles. L'objectif est double : «faire en sorte que les marchés du travail novateurs et dynamiques qui sous-tendent la compétitivité de l'Union s'inscrivent dans un cadre qui offre une protection de base à tous les travailleurs, garantit aux employeurs des gains de productivité à plus long terme et permet la convergence vers de meilleures conditions de vie et de travail dans toute I'Union ». Les directives WLB et TPWC sont susceptibles, ensemble, de créer un environnement de travail plus propice au soutien des travailleurs ayant des responsabilités familiales

On peut toutefois se demander dans quelle mesure l'article 9 vient soutenir les parents et les aidants. Cette disposition ne crée pas un droit légal fort et exécutoire. En effet, ce dernier reste formulé comme un droit de demander un aménagement des conditions de travail, plutôt que comme un droit de l'obtenir, de sorte que les employeurs sont simplement tenus d'examiner ces demandes et d'y répondre en tenant compte à la fois de leurs besoins

41 M. Bell, " EU Equality Law and Precarious Work», in U. Belavusau et K. Henrard (eds), EU AntiDiscrimination Law Beyond Gender, Hart Publishing, 2019, p. 75. Voir également F. Carmichael et S. Charles, "The Opportunity Costs of Informal Care: Does Gender Matter », Journal of Health Economics, 2003, p. 781.

42 S. Fredman, "Women at Work: The Broken Promises of Flexicurity », Industrial Law Journal, 2004, p. 299.

43 Clause 6(1) de la directive 2010/18.

44 Directive 2019/1152. 
et de ceux des travailleurs. Autre faiblesse de l'article 9 : il est de facto structuré de manière à répondre principalement aux besoins des salariés. Les travailleurs n'ayant que peu de contrôle sur leur travail, tels que les agents de nettoyage et les infirmiers, peuvent ne pas être en mesure de négocier les aménagements de travail dont ils ont besoin et ne sont probablement pas en mesure de faire du télétravail. Il est aussi fort probable que lorsqu'ils parviennent à réduire leur temps de travail, cela entraîne un coût financier.

\section{E - Mesures de PROTECTION DES TRAVAILlEuRS}

Outre l'introduction et le renforcement des quatre droits susmentionnés, la directive contient des dispositions visant à garantir l'efficacité de ces droits. À cette fin, l'article 10 prévoit le maintien des droits acquis par le travailleur au début du congé, ainsi que le droit de retrouver son poste de travail, ou un emploi équivalent, à la fin du congé. L'article 11 interdit tout traitement moins favorable au motif qu'un travailleur a demandé à bénéficier ou a fait usage des droits prévus par la directive. Cette disposition est complétée par l'article 12 qui interdit le licenciement et toute mesure préparatoire au licenciement d'un travailleur au motif qu'il a demandé ou fait valoir l'un des droits prévus par la directive WLB. Si cette protection est désormais courante dans les directives sur l'égalité, la directive WLB va plus loin en interdisant expressément toute discrimination au motif qu'un travailleur a "demandé » un droit. De cette manière, la directive WLB vise à protéger le souhait du travailleur d'user de ses droits et à faire en sorte qu'il n'ait pas peur de les exercer effectivement.

Ces dispositions sont importantes et il faut se féliciter qu'elles soient désormais des « clauses types». Cependant, elles ne sont pas suffisantes pour saisir la nature des difficultés auxquelles les individus sont confrontés en termes de responsabilités familiales. Pour y parvenir et pour faciliter la réalisation des objectifs de la directive, les travailleurs devraient être protégés non seulement contre la discrimination et les licenciements pour avoir utilisé leurs congés et leurs droits, mais aussi contre la discrimination en raison de leurs responsabilités familiales ${ }^{45}$.

\section{Conclusion}

II est évident que la directive sur l'équilibre entre vie professionnelle et vie privée est une belle avancée, attendue depuis fort longtemps. Il est intéressant de noter qu'il s'agit du premier instrument législatif qui aborde explicitement l'équilibre entre vie professionnelle et vie privée en tant que concept autonome. II renforce la dimension sociale de l'UE et peut permettre de créer un environnement qui reconnaît enfin que s'occuper de ses proches est inévitable et fait partie intégrante de la vie des travailleurs. Autrement dit, cette directive inscrit explicitement les charges et responsabilités familiales à l'ordre du jour de l'UE. Elle offre également aux législateurs nationaux l'occasion de repenser leur cadre juridique national.

Il faut toutefois bien comprendre que la directive WLB n'est qu'une étape de plus dans un long parcours. Si elle est sans doute la plus importante à ce jour, elle n'est pas la dernière. La directive reconnaît les difficultés auxquelles sont confrontés les parents et les aidants qui travaillent, mais elle ne répond pas à leurs attentes.

45 E. Caracciolo di Torella, " Shaping and Re-Shaping the Caring Relationship in European Law: a EU Catalogue of Rights for Carers », Child and Family Law Quarterly, 2016, p. 261. 


\section{ANALYSE DE LA DIRECTIVE 2019/1158}

Il est impératif de poursuivre sur cette lancée et d'identifier des points qui doivent encore être examinés par le législateur:

- Premièrement, l'absence de mention de la situation des mères est regrettable. On peut comprendre que le législateur n'ait pas voulu aborder les droits qui ont entraîné le retrait de la proposition de révision de la directive sur les travailleuses enceintes, mais un instrument sur l'équilibre entre vie professionnelle et vie privée devrait faire expressément référence aux mères également.

- Deuxièmement, les droits sont, de facto, limités. Si, à première vue, le congé de paternité et le congé parental jettent les bases d'un cadre législatif plus solide en ce qui concerne les hommes et les responsabilités familiales, on constate toutefois, en y regardant de plus près, que les mères sont toujours considérées comme les principales responsables du foyer. Il est peu probable que le congé des aidants renforce la position de ces derniers. Leur situation est d'autant plus difficile que la définition des aidants utilisée dans la directive WLB est extrêmement restrictive et ne permet probablement pas de saisir la nature de nombreuses relations de prise en charge. Bien que la directive s'efforce de répondre aux besoins d'une société vieillissante ${ }^{46}$, cette interprétation restrictive revient en fait à favoriser les parents de jeunes enfants au détriment des aidants.

- Troisièmement, les formules souples de travail sont toujours présentées comme un simple droit qui s'exerce sur demande et non comme un droit en soi.

- Quatrièmement, il est difficile de savoir si les travailleurs les plus vulnérables pourront bénéficier de la directive. Le législateur a cherché à élargir l'éventail des travailleurs pouvant en bénéficier; mais il est crucial de voir comment la CJUE interprétera l'article 2. Le législateur a aussi clairement voulu reconnaître toute la diversité des familles contemporaines. Toutefois, si la directive reconnaît les familles homosexuelles et fluides de genre, elle ne reconnaît guère les familles monoparentales, qui sont souvent les plus vulnérables.

- Enfin, la directive offre une protection contre la discrimination pour l'utilisation des congés/ droits, mais n'offre pas de protection contre la discrimination fondée sur les responsabilités familiales.

Ce n'est que lorsque ces points seront traités normativement que les parents et les aidants seront véritablement protégés et soutenus.

46 Considérant 27, directive 2019/1158.

\section{EUGENIA CARACCIOLO DI TORELLA}

Professeur associé, Faculté de droit, Université de Leicester.

Thèmes de recherche : Égalité, équilibre entre vie professionnelle et vie privée, vulnérabilité et droit.

\section{Publications:}

$\sim$ E. Caracciolo di Torella, " Here we go again: the Court, the value of care and traditional roles within the family », Common Market Law Review, 2020, vol. 57, n`1.

$\sim$ E. Caracciolo di Torella (avec A. Masselot), Caring Responsibilities in European Law and Policy: Who Cares?, Routledge, 2020. 